PROCEEDINGS OF THE

AMERICAN MATHEMATICAL SOCIETY

Volume 137, Number 4, April 2009, Pages 1235-1237

S 0002-9939(08)09616-0

Article electronically published on October 1, 2008

\title{
SET-THEORETIC HIDA PROJECTORS
}

\author{
AVNER ASH \\ (Communicated by Ken Ono)
}

\begin{abstract}
In his work on ordinary $p$-adic modular forms, Hida defined certain idempotents in any commutative algebra of finite rank over the ring of integers in a finite extension of $\mathbb{Q}_{p}$. We generalize his construction in the context of maps of finite sets and their inverse limits.
\end{abstract}

\section{INTRODUCTION}

Let $p$ denote a rational prime number, $K$ a finite extension of $\mathbb{Q}_{p}$ and $\mathcal{O}$ the ring of integers in $K$. In many papers, Hida has considered modules $M$ of finite rank over $\mathcal{O}$ with a linear operator $U$ acting on $M$. The ordinary part of $M$ is defined to be the maximal submodule of $M$ on which $U$ acts invertibly. For example, $M$ may be a space of $p$-adic modular forms, or the cohomology of an arithmetic group.

Hida constructed a projector onto the ordinary part of $M$. He gave several different constructions, but as an example, consider the following lemma:

Lemma 1. Let $A$ be a commutative $\mathcal{O}$-algebra (with multiplicative identity) of finite rank over $\mathcal{O}$ and $x \in A$. Then the limit

$$
\lim _{n \rightarrow \infty} x^{n !}
$$

exists in $A$ and gives an idempotent of $A$.

This lemma may be found on page 201 of Hida's book 2. It readily provides a construction of projectors onto the ordinary part of $M$ if $A$ is taken to be the ring of endomorphisms of $M$ and $x=U$. The proof Hida gives involves some elementary algebra of $p$-adic rings and the decomposition of an endomorphism into a sum of its semisimple and nilpotent parts.

The purpose of this paper is to give a purely set-theoretic version of this lemma, which behaves well under inverse limits. One easily recovers Hida's lemma, and one hopes that this more general version may be useful in situations, such as that of 1, where $p$-adic analytic families of such rings $A$ are at issue. Also our version works where there is no underlying prime $p$.

Received by the editors November 6, 2007, and, in revised form, April 15, 2008.

2000 Mathematics Subject Classification. Primary 11F33; Secondary 11F75.

Key words and phrases. p-adic, ordinary, projector, idempotent.

The author wishes to thank the National Science Foundation for support of this research through NSF grant DMS-0455240.

(C)2008 American Mathematical Society Reverts to public domain 28 years from publication 


\section{HidA-STYLE PROJECTORS}

Theorem 2. Let $V$ be a nonempty (possibly infinite) set and $f: V \rightarrow V$ a set function such that $f(V)$ has cardinality $t<\infty$. Then:

(1) For any two positive multiples $m, n$ of $t$ !, $f^{m}=f^{n}$.

(2) If $c \geq t, f^{c !}$ is an idempotent in the monoid under composition of functions from $V$ to $V$.

(3) $e_{f}:=\lim _{n \rightarrow \infty} f^{n !}$ is well-defined and an idempotent.

Proof. The descending chain of subsets $V \supset f(V) \supset f^{2}(V) \supset \cdots$ must stabilize after at most $t$ steps. Hence we have $f: f^{t}(V) \rightarrow f^{t}(V)$ is an automorphism of a set with at most $t$ elements. Therefore $f^{t !}: f^{t}(V) \rightarrow f^{t}(V)$ is the identity map.

Now $m \geq t$ !, so that also $m \geq t$. Set $b=t$ ! $-t$. Then for some $\beta \geq 0$, we can write $m-t=b+\beta t$ !.

Now for any $v \in V, f^{m} v=f^{m-t} f^{t} v=f^{b+\beta t !} f^{t} v=f^{b+t} v$, which is independent of $m$. In particular, $\left(f^{c !}\right)^{2}=f^{2 c !}=f^{c !}$.

Also, $e_{f}=\lim _{n \rightarrow \infty} f^{n !}$ is well-defined and an idempotent, because $n \geq c \geq$ $t \Longrightarrow t !|c !| n ! \Longrightarrow f^{n !}=f^{c !}$.

Note that the finiteness of the image of $f$ in the hypothesis is necessary. For example, if $V=\mathbb{Z}$ and $f$ is the shift operator, $f(x)=x+1$, then $f^{n !}$ has no limit.

Corollary 3. Let $V$ be a nonempty projective limit of the sets $V_{i}$ with respect to the transition maps $r_{i j}: V_{i} \rightarrow V_{j}$. Suppose $f_{i}: V_{i} \rightarrow V_{i}$ are set functions that compile into a function

$$
f=\lim _{\leftarrow} f_{i}: V \rightarrow V .
$$

Assume $f_{i}\left(V_{i}\right)$ finite for every $i$ and let $e_{f_{i}}$ be the idempotent constructed in Theorem 2. Then the $e_{f_{i}}$ compile into a function

$$
e_{f}:=\lim _{\leftarrow} e_{f_{i}}: V \rightarrow V
$$

and $e_{f}$ is an idempotent, i.e. $e_{f} \circ e_{f}=e_{f}$.

Proof. By hypothesis, for any $i>j, r_{i j} \circ f_{i}=f_{j} \circ r_{i j}$. Therefore, for any positive $m, r_{i j} \circ f_{i}^{m}=f_{j}^{m} \circ r_{i j}$. It follows that $r_{i j} \circ e_{f_{i}}=e_{f_{j}} \circ r_{i j}$. Thus, $e_{f}$ is well-defined. Since each $e_{f_{i}}$ is an idempotent, so is $e_{f}$.

\section{Applications}

We indicate how to prove Lemma 1 from Corollary 3 , We write

$$
A=\lim _{\leftarrow} A / p^{m} A .
$$

If $x \in A$, let $\mu_{x}$ denote multiplication by $x$. For each $m \geq 1, A / p^{m} A$ is a finite set and the $\mu_{x}: A / p^{m} A \rightarrow A / p^{m} A$ compile into $\mu_{x}: A \rightarrow A$. Note that for any $t \geq 1$, $\mu_{x^{t}}=\left(\mu_{x}\right)^{t}$.

By the corollary,

$$
e:=\lim _{\leftarrow} \mu_{x^{n !}}: A \rightarrow A
$$

exists and is a well-defined idempotent. Hence

$$
e(1)=\lim _{\leftarrow} \mu_{x^{n !}}(1)=\lim _{\leftarrow} x^{n !},
$$

where the rightmost term is the limit in the sense of the inverse limit topology on $A$, and hence is an element of $A$. 
It remains to check that $e(1)$ is an idempotent. First note that for any $a \in A$,

$$
e(a)=\lim _{\leftarrow}\left(\mu_{x^{n !}}(a)\right)=\lim _{\leftarrow} x^{n !} a=\left(\lim _{\leftarrow} x^{n !}\right) a=e(1) a
$$

because multiplication by $a$ is continuous. Hence $e(1)^{2}=e(e(1))=e(1)$ since $e^{2}=e$.

As another example, consider

Corollary 4. Let $A$ be a commutative $\hat{\mathbb{Z}}$-algebra with multiplicative identity finitely generated over $\hat{\mathbb{Z}}$ and $x \in A$. Then the limit

$$
\lim _{n \rightarrow \infty} x^{n !}
$$

exists in $A$ and gives an idempotent of $A$.

The proof is the same as for Lemma 1 except that we replace the directed system $\left\{p^{m}\right\}$ by $\{d \geq 1\}$ ordered by divisibility.

\section{REFERENCES}

[1] Avner Ash and Glenn Stevens, p-adic deformations of automorphic cohomology, preprint, http://www2.bc.edu/ ashav/Papers/Ash-Stevens-0ct-07-DRAFT-copy.pdf.

[2] Haruzo Hida, Elementary theory of $L$-functions and Eisenstein series, London Mathematical Society Student Texts, 26, Cambridge Univ. Press, Cambridge (1993). MR1216135|(94j:11044)

Department of Mathematics, Boston College, Chestnut Hill, Massachusetts 02445

E-mail address: Avner.Ash@bc.edu 